QUARTERLY OF APPLIED MATHEMATICS

VOLUME LXVIII, NUMBER 1

MARCH 2010, PAGES 113-134

S 0033-569X(09)01141-9

Article electronically published on October 26, 2009

\title{
QUADRATIC MORAWETZ INEQUALITIES AND ASYMPTOTIC COMPLETENESS IN THE ENERGY SPACE FOR NONLINEAR SCHRÖDINGER AND HARTREE EQUATIONS
}

\author{
BY \\ JEAN GINIBRE (Laboratoire de Physique Théorique, Université de Paris XI, Bâtiment 210, \\ F-91405 ORSAY Cedex, France) \\ AND \\ GIORGIO VELO (Dipartimento di Fisica, Università di Bologna and INFN, Sezione di Bologna, \\ Italy) \\ Dedicated to Professor Walter Strauss on his 70th birthday
}

\begin{abstract}
Recently several authors have developed multilinear and in particular quadratic extensions of the classical Morawetz inequality. Those extensions provide (among other results) an easy proof of asymptotic completeness in the energy space for nonlinear Schrödinger equations in arbitrary space dimension and for Hartree equations in space dimension greater than two in the noncritical cases. We give a pedagogical review of the latter results.
\end{abstract}

1. Introduction. This paper is devoted to the exposition of an elementary subset of some recent results bearing on scattering theory for the nonlinear Schrödinger (NLS) equation

$$
i \partial_{t} u=-(1 / 2) \Delta u+g(\rho) u
$$

in $n$ space dimensions, where $u$ is a complex-valued function defined in space time $\mathbb{R}^{n+1}$, $\rho=|u|^{2}$ and $g$ is a real-valued function of $\rho$, typically a sum of powers

$$
g(\rho)=\lambda_{1} \rho^{\left(p_{1}-1\right) / 2}+\lambda_{2} \rho^{\left(p_{2}-1\right) / 2}
$$

with $1<p_{1}<p_{2}$ and $\lambda_{1}, \lambda_{2} \in \mathbb{R}$. We also present a straightforward extension of those results to the Hartree equation (1.1) with

$$
g(\rho)=V \star \rho,
$$

Received July 3, 2008 and, in revised form, October 7, 2008.

2000 Mathematics Subject Classification. Primary 35P25; Secondary 35B40, 35Q40, 35Q55.

First author's sponsoring institution: Unité Mixte de Recherche (CNRS) UMR 8627.

E-mail address: Jean.Ginibre@th.u-psud.fr

E-mail address: Velo@bo.infn.it 
where $V$ is a real even function of the space variable and $\star$ denotes the convolution in $\mathbb{R}^{n}$. The first main question of scattering theory is the existence of the wave operators, namely the construction of solutions that behave asymptotically in time as solutions of the free Schrödinger equation, namely such that

$$
u(t) \sim U(t) u_{+} \quad \text { for } t \rightarrow \infty
$$

(and the analogue for $t \rightarrow-\infty$ ), where

$$
U(t)=\exp (i(t / 2) \Delta) .
$$

The second main question of scattering theory is asymptotic completeness (AC), simply called "scattering" in some of the recent literature, and consists in proving that all solutions of the relevant equation, in a suitable functional framework, behave asympotically as solutions of the free Schrödinger equation, namely satisfy (1.4) and the analogue for $t \rightarrow-\infty$. Of special interest is the case of finite energy solutions of (1.1), namely of solutions in $L^{\infty}\left(\mathbb{R}, H^{1}\right)$. An essential tool in the proof of $\mathrm{AC}$ for such solutions is the Morawetz inequality, first derived for the nonlinear Klein-Gordon (NLKG) equation [17] and then extended to the NLS equation [15]. That inequality was applied to prove AC first for the NLKG equation and then for the NLS equation in space dimension $n \geq 3$ in seminal papers by Morawetz and Strauss [18, and by Lin and Strauss [15, for slightly more regular solutions. The case of general finite energy solutions in space dimension $n \geq 3$ was treated later in [12] for NLS equations and in [13] for Hartree equations. The treatment was then improved for the NLS equation in [19] which covers in addition the more difficult cases $n=1,2$, as well as the case of the NLKG equation, and for the Hartree equation in [20].

More recently, several groups of authors have studied the more difficult problem of extending some of the previous results, in particular the proof of existence of global solutions and the proof of AC ("scattering"), on the one hand to the case of critical interactions, and on the other hand to the case of subenergy solutions, namely of solutions of intermediate regularity between $L^{2}$ and $H^{1}$. An important tool in some of those works is a new version of the Morawetz inequality, of a multilinear and in particular bilinear or quadratic type. That inequality has appeared in various forms in the literature and seems to have now stabilized to a simple form (11, 3]-10, [14, 21], 23]-25] and the references therein quoted). Leaving aside the difficult problems arising for critical interactions and/or for subenergy solutions, that new inequality provides a unified proof of AC for noncritical NLS in the energy space for all space dimensions, as well as for the Hartree equation for $n \geq 3$. That proof is much simpler than the previous ones. The present paper is devoted to an exposition of that new quadratic Morawetz inequality and of its application to the proof of AC for the NLS and Hartree equations in the energy space in noncritical situations. That result for noncritical NLS appears as a by product for $n=1$ in [5], for $n=2$ in [4] and for $n \geq 3$ in [24, which is mostly devoted to the critical cases. In Section 2, we first derive the quadratic Morawetz identity and we deduce therefrom the basic estimate that leads to the proof of AC. The formal proof of the identity is formulated in terms of conservation laws, in the spirit of 21. In Section 3, we exploit the previous estimate to prove AC. We treat the case of the NLS equation in 
some detail, and we give the modifications needed for the Hartree equation. The result applies to $L^{2}$ supercritical and $H^{1}$ subcritical nonlinearities. Some peripheral results are collected in the appendices. In Appendix 1, we give an estimate which points to the usefulness of the Morawetz inequality at lower regularity levels than $H^{1}$, in particular at the level of $H^{1 / 2}$. In Appendix 2, we exploit the point of view of conservation laws to derive a quadratic identity for the NLKG equation. That identity however does not lead to estimates because of a lack of positivity. In Appendix 3, we rewrite the original Morawetz inequality for the NLS equation in a form which exhibits its relation to the quadratic identity derived in Section 2. In Appendix 4, we justify the formal computation of Section 2 by a suitable limiting procedure.

We conclude this Introduction by giving some notation and estimates which will be used freely throughout this paper. For any integer $n \geq 1$, for any $r, 1 \leq r \leq \infty$, we denote by $\|\cdot\|_{r}$ the norm in $L^{r} \equiv L^{r}\left(\mathbb{R}^{n}\right)$, by $\bar{r}$ the conjugate exponent defined by $1 / r+1 / \bar{r}=1$, and we define $\delta(r) \equiv n / 2-n / r$. We denote by $\langle\cdot, \cdot\rangle$ the scalar product in $L^{2}$. We shall use the Sobolev spaces $\dot{H}_{r}^{\sigma} \equiv \dot{H}_{r}^{\sigma}\left(\mathbb{R}^{n}\right)$ and $H_{r}^{\sigma} \equiv H_{r}^{\sigma}\left(\mathbb{R}^{n}\right)$ defined for $0 \leq \sigma<\infty$ and $1<r<\infty$ by

$$
\begin{gathered}
\dot{H}_{r}^{\sigma}=\left\{u:\left\|u ; \dot{H}_{r}^{\sigma}\right\| \equiv\left\|\omega^{\sigma} u\right\|_{r}<\infty\right\}, \\
H_{r}^{\sigma}=\left\{u:\left\|u ; H_{r}^{\sigma}\right\| \equiv\left\|\langle\omega\rangle^{\sigma} u\right\|_{r}<\infty\right\},
\end{gathered}
$$

where $\omega=(-\Delta)^{1 / 2}$ and $\langle\cdot\rangle=\left(1+|\cdot|^{2}\right)^{1 / 2}$. The subscript $r$ will be omitted if $r=2$. For any interval $I$ of $\mathbb{R}$, for any Banach space $X$, we denote by $\mathscr{C}(I, X)$ the space of continuous functions from $I$ to $X$ and, for $1 \leq q \leq \infty$, by $L^{q}(I, X)$ (resp. $L_{l o c}^{q}(I, X)$ ) the space of measurable functions from $I$ to $X$ such that $\|u(\cdot) ; X\| \in L^{q}(I)$ (resp. $\left.\in L_{\text {loc }}^{q}(I)\right)$.

We introduce the following definition. A pair of exponents $(q, r)$ is admissible if $0 \leq 2 / q=\delta(r)=\delta$ and $\delta \leq 1 / 2$ for $n=1, \delta<1$ for $n=2$ and $\delta \leq 1$ for $n \geq 3$. Then the well-known Strichartz estimates take the form :

Lemma 1.1. Let $U(t)$ be given by (1.5). Then

(1) For any admissible pair $(q, r)$

$$
\left\|U(t) v ; L^{q}\left(\mathbb{R}, L^{r}\right)\right\| \leq C\|v\|_{2} .
$$

(2) For any admissible pairs $\left(q_{i}, r_{i}\right), i=1,2$, and for any interval $I$ of $\mathbb{R}$,

$$
\left\|\int_{I \cap\left\{t^{\prime}: t^{\prime} \leq t\right\}} d t^{\prime} U\left(t-t^{\prime}\right) f\left(t^{\prime}\right) ; L^{q_{1}}\left(I, L^{r_{1}}\right)\right\| \leq C\left\|f ; L^{\bar{q}_{2}}\left(I, L^{\bar{r}_{2}}\right)\right\|
$$

where the constant $C$ is independent of $I$.

Lemma 1.1 suggests studying the Cauchy problem for the equation (1.1) in spaces of the following type. Let $I$ be an interval of $\mathbb{R}$. We define

$$
X_{(l o c)}(I)=\left\{u: u \in \mathscr{C}\left(I, L^{2}\right) \text { and } u \in L_{l o c}^{q}\left(I, L^{r}\right) \text { for all admissible }(q, r)\right\}
$$

and

$$
X_{(l o c)}^{1}(I)=\left\{u: u, \nabla u \in X_{(l o c)}(I)\right\}
$$


2. Quadratic Morawetz inequalities. In this section we derive the quadratic Morawetz identity for the NLS and Hartree equations and we deduce therefrom the basic estimates that lead to the proof of asymptotic completeness in the energy space for those equations. We begin with a formal derivation of the quadratic Morawetz identity for the NLS equation, assuming sufficient smoothness and decay at infinity of the solutions to give a meaning to the calculation and in particular to the integrations by parts. The underlying algebraic structure is a pair of related conservation laws:

$$
\begin{gathered}
\partial_{t} \rho+\nabla \cdot j=0, \\
\partial_{t} j+\nabla \cdot T=0 .
\end{gathered}
$$

The first one is a scalar conservation law with scalar density $\rho$ and vector currrent $j$, the second one is a vector conservation law with vector density $j$ and second rank tensor current $T$, and the two laws are related by the fact that the current $j$ of the first one is at the same time the density of the second one. That situation occurs for the NLS equation and with a minor modification for the Hartree equation, as we shall review in this paper. It also occurs for any space time translation invariant system with a symmetric energy momentum tensor, with $\rho$ and $j$ being respectively the energy and momentum densities, and in particular for a class of NLKG equations, for which however it does not lead to useful estimates because of a lack of positivity (see Appendix 2 for the relevant formal calculation in that case). One could also consider the more general situation of two unrelated conservation laws, but that does not seem to be useful in the present case. Now let $h$ be a sufficiently regular real even function defined in $\mathbb{R}^{n}$. The starting point is the auxiliary quantity (which will be mostly forgotten at the end)

$$
J=(1 / 2)\langle\rho, h \star \rho\rangle .
$$

From (2.1) (2.2) and with two integrations by parts, it follows that

$$
\begin{aligned}
M & \equiv \partial_{t} J=-\langle\rho, h \star \nabla \cdot j\rangle=-\langle\rho, \nabla h \star j\rangle, \\
\partial_{t} M & =\partial_{t}^{2} J=\langle\nabla \cdot j, \nabla h \star j\rangle+\langle\rho, \nabla h \star \nabla \cdot T\rangle \\
& =-\left\langle j, \nabla^{2} h \star j\right\rangle+\left\langle\rho, \nabla^{2} h \star T\right\rangle,
\end{aligned}
$$

where $\nabla^{2} h$ is the second rank tensor $\nabla_{k} \nabla_{\ell} h$ and contractions are performed in the obvious way. The quadratic Morawetz identity is then the identity

$$
\partial_{t} M=-\partial_{t}\langle\rho, \nabla h \star j\rangle=-\left\langle j, \nabla^{2} h \star j\right\rangle+\left\langle\rho, \nabla^{2} h \star T\right\rangle .
$$

We now consider the NLS equation

$$
i \partial_{t} u=-(1 / 2) \Delta u+g u,
$$

where $g=g(\rho)$ is a real function of $\rho=|u|^{2}$. That equation is the Euler-Lagrange equation with Lagrangian density

$$
L(u)=-\operatorname{Im} \bar{u} \partial_{t} u-(1 / 2)|\nabla u|^{2}-G(\rho),
$$

where

$$
G(\rho)=\int_{0}^{\rho} d \rho^{\prime} g\left(\rho^{\prime}\right)
$$


The basic structure of (2.1) and (2.2) is realized with $\rho=|u|^{2}$ and

$$
j=\operatorname{Im} \bar{u} \nabla u,
$$

and (2.1) is the conservation law of the mass (or charge). The mass current $j$ turns out to be the momentum density, and (2.2) becomes the momentum conservation law. In fact the energy momentum tensor $\widetilde{T}$ is given by

$$
\left\{\begin{array}{l}
\widetilde{T}_{0 \ell}=2 \operatorname{Re} \frac{\partial L}{\partial\left(\partial_{t} u\right)} \nabla_{\ell} u=-\operatorname{Im} \bar{u} \nabla_{\ell} u=-j_{\ell}, \\
\widetilde{T}_{k \ell}=2 \operatorname{Re} \frac{\partial L}{\partial\left(\nabla_{k} u\right)} \nabla_{\ell} u-\delta_{k \ell} L=-\operatorname{Re} \nabla_{k} \bar{u} \nabla_{\ell} u-\delta_{k \ell} L
\end{array}\right.
$$

and (2.2) coincides (up to sign) with the conservation law

$$
\partial_{t} \widetilde{T}_{0 \ell}+\nabla_{k} \widetilde{T}_{k \ell}=0
$$

with $T_{k \ell}=-\widetilde{T}_{k \ell}$. For $u$ a solution of $(2.7), L(u)$ reduces to

$$
L(u)=-(1 / 4) \Delta \rho+\rho g(\rho)-G(\rho)
$$

so that

$$
T_{k \ell}=\operatorname{Re} \nabla_{k} \bar{u} \nabla_{\ell} u-\delta_{k \ell}((1 / 4) \Delta \rho-\rho g+G) .
$$

The conservation law (2.2) then holds with $j$ and $T$ defined by (2.10) and (2.14). Namely

$$
\partial_{t} j=-\nabla \cdot \operatorname{Re} \nabla \bar{u} \nabla u+\nabla((1 / 4) \Delta \rho-\rho g+G),
$$

which can of course be obtained by a direct computation using (2.7). Substituting (2.10) and (2.14) or (2.15) into (2.6) yields

$$
\begin{gathered}
\partial_{t} M=\langle\rho, \Delta h \star(-(1 / 4) \Delta \rho+\rho g-G)\rangle-\left\langle j, \nabla^{2} h \star j\right\rangle \\
+\left\langle\rho, \nabla^{2} h \star \nabla \bar{u} \nabla u\right\rangle,
\end{gathered}
$$

where we have used the symmetry of $\nabla^{2} h$ to eliminate the real part condition in the last term. On the other hand

$$
\begin{aligned}
\left\langle j, \nabla^{2} h \star j\right\rangle & =\left\langle\bar{u} \nabla u, \nabla^{2} h \star \bar{u} \nabla u\right\rangle-\left\langle\operatorname{Re} \bar{u} \nabla u, \nabla^{2} h \star \operatorname{Re} \bar{u} \nabla u\right\rangle \\
& =\left\langle\bar{u} \nabla u, \nabla^{2} h \star \bar{u} \nabla u\right\rangle-(1 / 4)\left\langle\nabla \rho, \nabla^{2} h \star \nabla \rho\right\rangle
\end{aligned}
$$

so that

$$
\partial_{t} M=(1 / 2)\langle\nabla \rho, \Delta h \star \nabla \rho\rangle+\langle\rho, \Delta h \star(\rho g-G)\rangle+R,
$$

where we have used the fact that

$$
-\langle\rho, \Delta h \star \Delta \rho\rangle=\left\langle\nabla \rho, \nabla^{2} h \star \nabla \rho\right\rangle=\langle\nabla \rho, \Delta h \star \nabla \rho\rangle
$$

by integration by parts, and where

$$
\begin{aligned}
R & =\left\langle\bar{u} u, \nabla^{2} h \star \nabla \bar{u} \nabla u\right\rangle-\left\langle\bar{u} \nabla u, \nabla^{2} h \star \bar{u} \nabla u\right\rangle \\
& =(1 / 2) \int d x d y(\bar{u}(x) \nabla \bar{u}(y)-\bar{u}(y) \nabla \bar{u}(x)) \nabla^{2} h(x-y) \\
& \times(u(x) \nabla u(y)-u(y) \nabla u(x)) .
\end{aligned}
$$


Integrating (2.18) over time in an interval $\left[t_{1}, t_{2}\right]$ yields

$$
\begin{aligned}
& \int_{t_{1}}^{t_{2}} d t\{(1 / 2)\langle\nabla \rho, \Delta h \star \nabla \rho\rangle+\langle\rho, \Delta h \star(\rho g-G)\rangle+R\} \\
& \quad=-\left.\langle\rho, \nabla h \star \operatorname{Im} \bar{u} \nabla u\rangle\right|_{t_{1}} ^{t_{2}} .
\end{aligned}
$$

That identity will yield useful estimates if $\nabla h \in L^{\infty}$ and if $\nabla^{2} h$ is nonnegative as a matrix. Under the latter assumption, $R$ is nonnegative, the first term in the integrand is positive, and the second term is nonnegative if $\rho g-G \geq 0$.

We now consider a representative situation where the previous formal computations can be made rigorous. We take $h(x)=|x|$, so that

$$
\left\{\begin{array}{l}
\nabla h \quad=|x|^{-1} x, \\
\nabla^{2} h \quad=|x|^{-1}\left(\mathbb{1}-|x|^{-2} x \otimes x\right), \Delta h=(n-1)|x|^{-1} \quad \text { for } n \geq 2, \\
\nabla^{2} h=\Delta h=2 \delta(x) \quad \text { for } n=1 .
\end{array}\right.
$$

In that case

$$
\begin{gathered}
\langle\nabla \rho, \Delta h \star \nabla \rho\rangle=c\left\langle\nabla \rho, \omega^{1-n} \nabla \rho\right\rangle=c\left\|\rho ; \dot{H}^{(3-n) / 2}\right\|^{2}, \\
\langle\rho, \Delta h \star(\rho g-G)\rangle=c\left\langle\rho, \omega^{1-n}(\rho g-G)\right\rangle,
\end{gathered}
$$

where $\omega=(-\Delta)^{1 / 2}$ and $c$ is a constant depending only on $n[22$.

We take for $g$ a sum of two powers

$$
g(\rho)=\lambda_{1} \rho^{\left(p_{1}-1\right) / 2}+\lambda_{2} \rho^{\left(p_{2}-1\right) / 2}
$$

with $\lambda_{1}, \lambda_{2} \in \mathbb{R}$, so that

$$
\begin{gathered}
G(\rho)=2 \lambda_{1}\left(p_{1}+1\right)^{-1} \rho^{\left(p_{1}+1\right) / 2}+2 \lambda_{2}\left(p_{2}+1\right)^{-1} \rho^{\left(p_{2}+1\right) / 2}, \\
\rho g(\rho)-G(\rho)=\lambda_{1} \frac{p_{1}-1}{p_{1}+1} \rho^{\left(p_{1}+1\right) / 2}+\lambda_{2} \frac{p_{2}-1}{p_{2}+1} \rho^{\left(p_{2}+1\right) / 2},
\end{gathered}
$$

with $1 \leq p_{1}<p_{2}$. More general $g$ can be easily accommodated. For $H^{1}$ subcritical powers, the Cauchy problem for NLS is well known to be locally well posed in $X_{l o c}^{1}$ for initial data in $H^{1}$ and possibly globally well posed [2].

Proposition 2.1. Let $h(x)=|x|$ and let $g$ be defined by (2.25) with $1 \leq p_{1}<p_{2}$ and $p_{2}<1+4 /(n-2)$ for $n \geq 3$. Let $I$ be an interval and let $u \in X_{l o c}^{1}(I)$ be a solution of the NLS equation (2.7). Then

(1) The identity (2.21) holds for any $t_{1}, t_{2} \in I$.

(2) Let in addition $\lambda_{1}, \lambda_{2} \geq 0$ (so that $u \in X_{\text {loc }}^{1}(\mathbb{R}) \cap L^{\infty}\left(\mathbb{R}, H^{1}\right)$ ). Then $u$ satisfies the estimate

$$
\left\|\rho ; L^{2}\left(\mathbb{R}, \dot{H}^{(3-n) / 2}\right)\right\|^{2}+\int d t\left\langle\rho, \omega^{1-n}(\rho g-G)\right\rangle \leq C\|u\|_{2}^{3}\left\|u ; L^{\infty}\left(\mathbb{R}, H^{1}\right)\right\| .
$$

In particular $\rho \in L^{2}\left(\mathbb{R}, \dot{H}^{(3-n) / 2}\right)$. 
Sketch of proof. The proof of part (1) consists in making the previous formal computation rigorous under the available regularity properties by introducing suitable cutoffs and eliminating them by a limiting procedure. This is done in Appendix 4. At this level of regularity, one easily checks that all the terms in the identity are already well defined in the differential form (2.18). Actually, by (2.23) and Sobolev inequalities,

$$
\begin{aligned}
\langle\nabla \rho, \Delta h \star \nabla \rho\rangle & \leq C\|u\|_{r}^{2}\|\nabla u\|_{2}^{2} & \text { with } n / 2-n / r=1 / 2 \\
& \leq C\left\|u ; \dot{H}^{1 / 2}\right\|^{2}\|\nabla u\|_{2}^{2} & \text { for } n \geq 2 .
\end{aligned}
$$

Similarly, for $g$ a single power $p$,

$$
\begin{gathered}
|\langle\rho, \Delta h \star(\rho g-G)\rangle| \leq C\|u\|_{p+1}^{p+1}\left\|\omega^{1-n}|u|^{2}\right\|_{\infty} \\
\leq C\|u\|_{p+1}^{p+1}\|u\|_{r_{+}}\|u\|_{r_{-}} \quad \text { for } n \geq 2
\end{gathered}
$$

with $n / r_{ \pm}=n / 2-1 / 2 \pm \varepsilon$. Furthermore $R=0$ for $n=1$, while for $n \geq 2, R$ is the sum of terms of the type $\left\langle\bar{u} \nabla u, \nabla^{2} h \star \bar{u} \nabla u\right\rangle$ which are estimated as in (2.29), and $\left\langle\bar{u} u, \nabla^{2} h \star \nabla \bar{u} \nabla u\right\rangle$ which are estimated by

$$
\begin{aligned}
& \left|\left\langle\bar{u} u, \nabla^{2} h \star \nabla \bar{u} \nabla u\right\rangle\right| \leq C\left\langle|u|^{2}, \omega^{1-n}|\nabla u|^{2}\right\rangle \\
& \quad \leq C\|\nabla u\|_{2}^{2}\left\|\omega^{1-n}|u|^{2}\right\|_{\infty} \leq C\|\nabla u\|_{2}^{2}\|u\|_{r_{+}}\|u\|_{r_{-}} .
\end{aligned}
$$

Finally the right-hand side of (2.21) is estimated by

$$
|\langle\rho, \nabla h \star \operatorname{Im} \bar{u} \nabla u\rangle| \leq\|\nabla h\|_{\infty}\|u\|_{2}^{3}\|\nabla u\|_{2} .
$$

Part (2) follows from (2.21) by taking the limit $t_{1} \rightarrow-\infty, t_{2} \rightarrow \infty$, from (2.23) and (2.24), from the positivity of $\rho g-G$ and of $R$, and from (2.32).

We now briefly sketch some further developments along the previous lines. First the formal computation leading to (2.18) can easily be extended to yield a bilinear Morawetz identity for two solutions of the NLS equation. Actually the identity (2.18) can also be arrived at by applying the original Morawetz identity [15] to a suitable tensor product of two solutions of (2.7). Therefore let $u_{i}, i=1,2$, be two solutions of (2.7), let $\rho_{i}, j_{i}$, $T_{i}$ be the associated density, current and tensor $T$, and let $g_{i}=g\left(\rho_{i}\right), G_{i}=G\left(\rho_{i}\right)$. We start from

$$
J=(1 / 2)\left\langle\rho_{1}, h \star \rho_{2}\right\rangle
$$

so that

$$
\begin{gathered}
M \equiv \partial_{t} J=-(1 / 2)\left(\left\langle\rho_{1}, \nabla h \star j_{2}\right\rangle+\left\langle\rho_{2}, \nabla h \star j_{1}\right\rangle\right), \\
\partial_{t} M=\partial_{t}^{2} J=-\left\langle j_{1}, \nabla^{2} h \star j_{2}\right\rangle+(1 / 2)\left(\left\langle\rho_{1}, \nabla^{2} h \star T_{2}\right\rangle+\left\langle\rho_{2}, \nabla^{2} h \star T_{1}\right\rangle\right) .
\end{gathered}
$$

Substituting (2.10) and (2.14) into (2.35) and proceeding as before, we obtain

$$
\begin{gathered}
\partial_{t} M=(1 / 2)\left\{\left\langle\nabla \rho_{1}, \Delta h \star \nabla \rho_{2}\right\rangle+\left\langle\rho_{1}, \Delta h \star\left(\rho_{2} g_{2}-G_{2}\right)\right\rangle\right. \\
\left.+\left\langle\rho_{2}, \Delta h \star\left(\rho_{1} g_{1}-G_{1}\right)\right\rangle\right\}+R,
\end{gathered}
$$


where now

$$
\begin{aligned}
R & =(1 / 2)\left\{\left\langle\bar{u}_{1} u_{1}, \nabla^{2} h \star \nabla \bar{u}_{2} \nabla u_{2}\right\rangle+(1 \leftrightarrow 2)\right\}-\left\langle\bar{u}_{1} \nabla u_{1}, \nabla^{2} h \star \bar{u}_{2} \nabla u_{2}\right\rangle \\
& =(1 / 2) \int d x d y\left(\bar{u}_{1}(x) \nabla \bar{u}_{2}(y)-\bar{u}_{2}(y) \nabla \bar{u}_{1}(x)\right) \nabla^{2} h(x-y) \\
& \times\left(u_{1}(x) \nabla u_{2}(y)-u_{2}(y) \nabla u_{1}(x)\right) .
\end{aligned}
$$

The identity (2.36) is the bilinear version of (2.18). Remarkably enough, $R$ is still nonnegative in that case if $\nabla^{2} h$ is a nonnegative matrix. On the other hand, for a repulsive (defocusing) $g$, the terms in (2.36) containing $g$ are also nonnegative, while the first term in the bracket is positive for $n \geq 3$ (but in general not for $n=1,2$ ), so that in that case (2.36) yields some bilinear estimates. Whether such estimates can be useful remains to be seen.

A second further development of the previous calculation consists in using for $h$ functions other than $|x|$. For instance one can take $h(x)=|\theta \cdot x|$ for $\theta \in S^{n-1}$ and more generally $h(x)=|P x|$ for $P$ the orthogonal projection on a generic $k$-dimensional plane in $\mathbb{R}^{n}$. The first choice naturally leads to an estimate of the Radon transform of $\rho$ [21]. One can also take advantage of the fact that the derivation of (2.18) involves mainly two integrations by parts from $h$ to $\nabla^{2} h$ in order to treat the case of a domain $\Omega \subset \mathbb{R}^{n}$, typically the complement of a convex (or at least star-shaped) compact subset of $\mathbb{R}^{n}$. One then obtains identities similar to (2.18) with additional surface terms, from which one can derive estimates of solutions in $\Omega[21]$.

A third possible development consists in extending the estimates of Proposition 2.1, part (2) to the case of attractive (focusing) interactions $g$ and of small solutions. We consider for illustration the case of a single power

$$
g(\rho)=-\rho^{(p-1) / 2}
$$

in dimension $n=1$. In that case, $(2.18)$ becomes (remember that $R=0$ for $n=1$ )

$$
\partial_{t} M=\|\nabla \rho\|_{2}^{2}-\frac{p-1}{p+1} \int d x \rho^{(p+3) / 2} .
$$

By Sobolev inequalities, we estimate

$$
\|\rho\|_{(p+3) / 2}^{(p+3) / 2} \leq C\|\nabla \rho\|_{2}^{2}\|\rho\|_{(p-1) / 4}^{(p-1) / 2} \leq C\|\nabla \rho\|_{2}^{2}\left\|u ; \dot{H}^{\sigma_{c}}\right\|^{p-1},
$$

where $\sigma_{c}=1 / 2-2 /(p-1)$ is the value of $\sigma$ for which $g$ given by $(2.38)$ is $\dot{H}^{\sigma}$ critical, provided $\sigma_{c} \geq 0$, namely $p \geq 5$. Therefore

$$
\partial_{t} M \geq\|\nabla \rho\|_{2}^{2}\left(1-C\left\|u ; \dot{H}^{\sigma_{c}}\right\|^{p-1}\right),
$$

so that (2.18) again yields an a priori estimate of $\rho$ in $L^{2}\left(\mathbb{R}, \dot{H}^{1}\right)$ provided $M$ is controlled and provided $u$ is small in $L^{\infty}\left(\mathbb{R}, \dot{H}^{\sigma_{c}}\right)$. The latter condition can be realized for energy solutions by taking some initial data $u_{0}$ small in $L^{2}$ if $\sigma_{c}=0$, namely $p=5$, and $u_{0}$ small in $H^{1}$ if $p>5$. That smallness condition is of the same type as that occurring in the proof of boundedness of the $H^{1}$ norm from the energy conservation law which is used in the standard proof of globalization in $H^{1}$. 
A similar situation can occur in higher space dimensions insofar as one can prove the estimate

$$
\left|\left\langle\rho, \omega^{1-n} \rho^{(p+1) / 2}\right\rangle\right| \leq C\left\|\rho ; \dot{H}^{(3-n) / 2}\right\|^{2}\left\|u ; \dot{H}^{\sigma_{c}}\right\|^{p-1},
$$

where again $\sigma_{c}=n / 2-2 /(p-1)$ is the critical Sobolev exponent corresponding to $p$, provided $\sigma_{c} \geq 0$, namely $p \geq 1+4 / n$, the $L^{2}$ critical value. The estimate (2.42) can easily be proved by the use of Sobolev inequalities for $n=2,3$ and $p$ not too large. We leave the investigation of that estimate for general $n$ and $p$ as an open question.

A last possible development consists in using the Morawetz inequality to prove global wellposedeness and possibly AC ("scattering") at a lower level of regularity than $H^{1}$, and that possibility has been extensively exploited. See, for instance, [3]-[5, [7]-[10], [16], 25] and the references therein quoted. In particular the right-hand side of (2.21) is controlled by the $H^{1 / 2}$ norm of $u$. For completeness we give a proof of that fact in Appendix 1 (see also [7] for the case $n \geq 3$ ).

We now turn to the Hartree equation (1.1) with $g$ given by (1.3). The formal computation is almost the same as for the NLS equation, except for the fact that, because of the nonlocality of the interaction, the equation is not Lagrangian. However the evolution equation of $j$ for the NLS equation takes the form

$$
\partial_{t} j=\text { kinetic terms }-\rho \nabla g,
$$

as follows in the same way as (2.15) from a computation which can be done without referring to the special form of $g$, so that (2.43) also holds for the Hartree equation (1.1) (1.3). Substituting (2.43) into $\partial_{t} M$ and using the fact that the kinetic terms are unchanged, we obtain

$$
\partial_{t} M=(1 / 2)\langle\nabla \rho, \Delta h \star \nabla \rho\rangle+\langle\rho, \nabla h \star(\rho \nabla(V \star \rho))\rangle+R,
$$

where $R$ is given by $(2.20)$ as before. Integrating (2.44) over time in an interval $\left[t_{1}, t_{2}\right]$ yields

$$
\begin{aligned}
& \int_{t_{1}}^{t_{2}} d t\{(1 / 2)\langle\nabla \rho, \Delta h \star \nabla \rho\rangle+\langle\rho, \nabla h \star(\rho \nabla(V \star \rho))\rangle+R\} \\
& \quad=-\left.\langle\rho, \nabla h \star \operatorname{Im} \bar{u} \nabla u\rangle\right|_{t_{1}} ^{t_{2}} .
\end{aligned}
$$

As in the case of the NLS equation, that identity will yield useful estimates if $\nabla h \in L^{\infty}$ and if $\nabla^{2} h$ is nonnegative as a matrix, so that $R$ is nonnegative, and if in addition the potential term in (2.45) is nonnegative. We now show that this is the case if $V$ is radial and nonincreasing. Assuming sufficient smoothness and decay at infinity for $V$, we obtain

$$
\begin{aligned}
P & \equiv\langle\rho, \nabla h \star(\rho \nabla(V \star \rho))\rangle=\int d x d y d z \rho(x) \nabla h(x-y) \rho(y) \nabla V(y-z) \rho(z) \\
& =(1 / 2) \int d x d y d z \rho(x) \rho(y) \rho(z) \nabla V(y-z)(\nabla h(x-y)-\nabla h(x-z)),
\end{aligned}
$$

where we have used the fact that $\nabla V$ is an odd function. In order to prove the positivity of that integral, it suffices to prove that for all $x, y$

$$
\nabla V(x) \cdot(\nabla h(x+y)-\nabla h(y)) \leq 0,
$$


where we have changed variables from $(y-z, x-y, x-z)$ to $(x, y, x+y)$. Now let $V(x)=v(|x|)$. The left-hand side of (2.47) can be written as

$$
\begin{aligned}
\int_{0}^{1} d \theta \nabla V(x) x \cdot \nabla^{2} h(y+\theta x) \\
\quad=\int_{0}^{1} d \theta|x|^{-1} v^{\prime}(|x|)(x \otimes x) \cdot \nabla^{2} h(y+\theta x) \leq 0,
\end{aligned}
$$

for nonpositive $v^{\prime}$ and nonnegative $\nabla^{2} h$.

We now give a proposition where we assume sufficient regularity of $V$ to ensure wellposedness in $H^{1}$ and to make the previous formal computation rigorous.

Proposition 2.2. Let $h=|x|$ and let $V \in L^{p_{1}}+L^{p_{2}}$, where

$$
p_{2} \geq 1 \quad, \quad n / 4<p_{2}<p_{1} \leq \infty .
$$

Let $I$ be an interval and let $u \in X_{l o c}^{1}(I)$ be a solution of the Hartree equation (1.1), (1.3). Then

(1) The identity (2.45) holds for any $t_{1}, t_{2} \in I$.

(2) Let in addition $V$ be radial nonincreasing (so that $V$ is nonnegative, possibly up to a harmless constant, and $\left.u \in X_{l o c}^{1}(\mathbb{R}) \cap L^{\infty}\left(\mathbb{R}, H^{1}\right)\right)$. Then $u$ satisfies the estimate

$$
\left\|\rho ; L^{2}\left(\mathbb{R}, \dot{H}^{(3-n) / 2}\right)\right\|^{2} \leq C\|u\|_{2}^{3}\left\|u ; L^{\infty}\left(\mathbb{R}, H^{1}\right)\right\| .
$$

Sketch of proof. The proof of part (1) follows the same pattern as that of Proposition 2.1. Here we simply verify that the Hartree potential term $P$ in $(2.45)$ is well defined at the available level of regularity. By the Hölder and Young inequalities, we estimate

$$
\begin{gathered}
|P| \equiv|\langle\rho, \nabla h \star(\rho(V \star \nabla \rho))\rangle| \leq\|\rho\|_{1}\|\nabla h\|_{\infty}\|\rho\|_{k / 2}\|V\|_{p}\|u\|_{k}\|\nabla u\|_{2} \\
=C\|\rho\|_{1}\|\nabla u\|_{2}\|u\|_{k}^{3}
\end{gathered}
$$

with $\delta(k) \equiv n / 2-n / k=n /(3 p)$. For the relevant values of $p$, one can take $\delta(k) \leq 1 / 4$ for $n=1, \delta(k)<1$ for $n=2, \delta(k) \leq 1$ for $n=3$ and for $n \geq 4$ if $p \geq n / 3$, so that $\|u\|_{k}$ is controlled by the $H^{1}$ norm of $u$ and $P$ is controlled in $L_{l o c}^{\infty}(I)$, namely at the differential level. For $n \geq 4$ and $n / 4 \leq p<n / 3$, we use the fact that $u \in L_{l o c}^{q}\left(I, L^{k}\right)$ with $2 / q=\delta(k)-1=n /(3 p)-1 \leq 1 / 3$, so that $u \in L_{l o c}^{6}\left(I, L^{k}\right)$ and therefore $P \in L_{l o c}^{2}(I)$.

Part (2) follows from (2.45) by taking the limit $t_{1} \rightarrow-\infty, t_{2} \rightarrow \infty$, from (2.23) and (2.32), and from the positivity of $P$ defined in (2.46) and of $R$ defined by (2.20).

3. Asymptotic completeness in the energy space. In this section we exploit the Morawetz estimates of Propositions 2.1 and 2.2 to derive asymptotic completeness in $H^{1}$ for the NLS and Hartree equations. We begin with the NLS equation for which we restrict our attention to a single power interaction

$$
g(\rho)=\lambda \rho^{(p-1) / 2} .
$$

We shall use the parameter $\sigma_{c}$ defined equivalently by

$$
\sigma_{c}=n / 2-2 /(p-1) \quad \text { or } \quad p-1=4 /\left(n-2 \sigma_{c}\right)
$$


so that $g$ given by (3.1) is $\dot{H}^{\sigma_{c}}$ critical. We shall assume $0<\sigma_{c}<1$ so that $g$ is $L^{2}$ supercritical and $H^{1}$ subcritical. The treatment extends in a trivial way to a sum of such powers and to more general $g$. The case of critical powers is much more complicated, and we refer to 24] for a treatment of that case in dimension $n \geq 3$. Some of the arguments can also be applied to solutions in $H^{\sigma}$ for $0<\sigma \leq 1$.

The main technical step is the following proposition.

Proposition 3.1. Let $g$ be defined by (3.1) with $0<\sigma_{c}<1\left(\sigma_{c}<1 / 2\right.$ for $\left.n=1\right)$. Let $u \in X_{\text {loc }}^{1}(\mathbb{R}) \cap L^{\infty}\left(\mathbb{R}, H^{1}\right)$ be a solution of the NLS equation (1.1), (3.1) such that $\rho=|u|^{2} \in L^{2}\left(\mathbb{R}, \dot{H}^{(3-n) / 2}\right)$. Then $u \in X^{1}(\mathbb{R})$.

REMARK 3.1. For repulsive (defocusing) interaction $g$, namely for $\lambda>0$, the Cauchy problem with initial data in $H^{1}$ is known to yield solutions satisfying the first assumption, and those solutions satisfy the condition on $\rho$ by Proposition 2.1. For attractive (focusing) interaction, the first assumption is satisfied for small data in $H^{1}$, and the assumption on $\rho$ can also be satisfied in some cases, for instance for $n=1$, and for $n=2,3$ and $p$ not too large, as discussed in the comments after Proposition 2.1 (see in particular (2.41)).

Proof. Let $I=\left[t_{0}, t_{1}\right]$ be an interval and $u_{0}=u\left(t_{0}\right)$. We start from the integral equation

$$
u(t)=U\left(t-t_{0}\right) u_{0}-i \int_{t_{0}}^{t} d t^{\prime} U\left(t-t^{\prime}\right) g\left(\rho\left(t^{\prime}\right)\right) u\left(t^{\prime}\right) .
$$

Using the Strichartz inequalities, we estimate in a standard way [2]

$$
\begin{aligned}
& \left\|u ; X^{1}(I)\right\| \leq C\left(\left\|u_{0}: H^{1}\right\|+\left\|g(\rho) u ; L^{\bar{q}}\left(I, H_{\bar{r}}^{1}\right)\right\|\right) \\
& \quad \leq C\left(\left\|u_{0} ; H^{1}\right\|+\left\|u ; X^{1}(I)\right\|\left\|u ; L^{k}\left(I, L^{\ell}\right)\right\|^{p-1}\right),
\end{aligned}
$$

where $1 / \bar{r}+1 / r=1 / \bar{q}+1 / q=1,(q, r)$ is an admissible pair, and

$$
\left\{\begin{array}{l}
2 / k=\left(n / 2-\sigma_{c}\right)(1-\delta), \\
n / \ell=\left(n / 2-\sigma_{c}\right) \delta,
\end{array}\right.
$$

where $\delta \equiv \delta(r)=n / 2-n / r$.

The main step of the proof consists in estimating $u$ in $L^{k}\left(L^{\ell}\right)$ by interpolation between the Morawetz quantity $\left\|\rho ; L^{2}\left(\dot{H}^{(3-n) / 2}\right)\right\|$ and some norm which is controlled by $\left\|u ; L^{\infty}\left(H^{1}\right)\right\|$, typically $\left\|u ; L^{\infty}\left(\dot{H}^{\sigma}\right)\right\|$ for some $\sigma, 0 \leq \sigma \leq 1$. For orientation, we first consider the homogeneity degree of the various norms involved, where the degree of $\left\|u ; L^{q}\left(\dot{H}_{r}^{\sigma}\right)\right\|$ is defined as $\sigma+\delta(r)-2 / q$, so that it reduces to $\sigma$ for admissible $(q, r)$. In particular the degree of $\left\|u ; L^{k}\left(L^{\ell}\right)\right\|$ is $\sigma_{c}$ by (3.5), that of $\left\|u ; L^{\infty}\left(\dot{H}^{\sigma}\right)\right\|$ is $\sigma$, and the degree $\sigma_{M}$ of the Morawetz quantity is obtained by comparing from the point of view of dimension

$$
\left\|\rho ; L^{2}\left(\dot{H}^{(3-n) / 2}\right)\right\| \sim\left\|u ; L^{\infty}\left(\dot{H}^{\sigma_{M}}\right)\right\|^{2}
$$

which gives

$$
1+n / 2+(n-3) / 2=2\left(n / 2-\sigma_{M}\right)
$$

and therefore $\sigma_{M}=1 / 4$. 
We have to combine information on $u$ and on $\rho$, which can be transformed into information bearing only on $u$ or only on $\rho$. We consider separately the cases $n \geq 2$ and $n=1$.

The case $\mathbf{n} \geq 2$. Here we work with $u$. The information on $\rho$ implies the following information on $u$ :

$$
\begin{cases}\rho \in L^{2}\left(\mathbb{R}, \dot{H}^{1 / 2}\right) \subset L^{2}\left(\mathbb{R}, L^{4}\right) \Leftrightarrow u \in L^{4}\left(\mathbb{R}, L^{8}\right) & \text { for } n=2, \\ \rho \in L^{2}\left(\mathbb{R}, L^{2}\right) \Leftrightarrow u \in L^{4}\left(\mathbb{R}, L^{4}\right) & \text { for } n=3, \\ \rho \in L^{2}\left(\mathbb{R}, \dot{H}^{(3-n) / 2}\right) \Rightarrow u \in L^{4}\left(\mathbb{R}, \dot{H}_{4}^{(3-n) / 4}\right) & \text { for } n \geq 4,\end{cases}
$$

where the last result follows from Lemma 5.6 in [24. We want to estimate $u$ in $L^{k}\left(I, L^{\ell}\right)$ with $k, \ell$ satisfying (3.5) for some $k<\infty$ and some $\delta$ with $0 \leq \delta<1$ (the value $\delta=1$ is excluded a priori for $n=2$, and by the condition $k<\infty$ for $n \geq 3$ ). From (3.5), we obtain

$$
2 / k+n / \ell=n / 2-\sigma_{c} .
$$

Conversely if $k, \ell$ satisfy (3.7) with $1 \leq k<\infty$ and $2 \leq \ell \leq \infty$, then $\delta$ defined by (3.5) satisfies $0 \leq \delta<1$, so that it suffices to consider (3.7). We estimate by Sobolev inequalities and by (3.6) that

$$
\left\|u ; L^{k}\left(I, L^{\ell}\right)\right\| \leq C\left\|u ; L^{4}\left(I, \dot{H}_{4}^{(3-n) / 4}\right)\right\|^{\theta}\left\|u ; L^{\infty}\left(I, \dot{H}^{\sigma}\right)\right\|^{1-\theta}
$$

(where $\dot{H}_{4}^{1 / 4}$ should be replaced by $L^{8}$ for $n=2$ according to (3.6)) for some $\sigma$ and $\theta$ with $0 \leq \sigma \leq 1$ and $0<\theta \leq 1$, such that

$$
\left\{\begin{array}{l}
2 / k=\theta / 2, \\
n / \ell=\theta(n / 2-3 / 4)+(1-\theta)(n / 2-\sigma),
\end{array}\right.
$$

so that

$$
2 / k+n / \ell=n / 2-\sigma_{c}=\theta(n / 2-1 / 4)+(1-\theta)(n / 2-\sigma)
$$

or equivalently

$$
\sigma_{c}=\theta / 4+(1-\theta) \sigma
$$

in accordance with the homogeneity argument given above. In addition for $n \geq 4$, the Sobolev inequality requires

$$
\theta(n-3) / 4 \leq(1-\theta) \sigma .
$$

For a given $\sigma_{c}$ with $0<\sigma_{c}<1$, it is therefore sufficient to find $\sigma$ and $\theta$ with $0 \leq \sigma \leq 1$ and $0<\theta \leq 1$, satisfying (3.10) and in addition (3.11), or equivalently

$$
\theta \leq 4 \sigma /(n-3+4 \sigma)
$$

for $n \geq 4$. One can make the following choices.

Case $\mathbf{n}=2$, 3. For $\sigma_{c}=1 / 4$, one can take $\theta=1$, and the norm in $L^{\infty}\left(\dot{H}^{\sigma}\right)$ is not needed. For $\sigma_{c} \neq 1 / 4$, the allowed values of $\sigma$ are given by

$$
0 \leq \sigma<\sigma_{c}<1 / 4 \quad \text { or } \quad 1 / 4<\sigma_{c}<\sigma \leq 1,
$$

with $\theta$ defined by (3.10). 
Case $\mathbf{n} \geq 4$. For $\sigma_{c}=1 / 4$, one must take $\sigma=1 / 4$ and one can take $\theta=(n-2)^{-1}$. For $\sigma_{c} \neq 1 / 4$, the allowed values of $\sigma$ are given by

$$
(0<) \sigma_{0} \leq \sigma<\sigma_{c}<1 / 4 \quad \text { or } \quad 1 / 4<\sigma_{c}<\sigma \leq \sigma_{0} \wedge 1
$$

where $\sigma_{0}$ is defined by (3.10) and (3.12) with equality, namely

$$
\sigma_{0}=\sigma_{c}(n-3) /\left(n-2-4 \sigma_{c}\right),
$$

with $\theta$ defined by (3.10).

We can now complete the proof of the proposition. Substituting (3.8) into (3.4) yields

$$
\left\|u ; X^{1}(I)\right\|\left(1-M_{1}\left\|\rho ; L^{2}\left(I, \dot{H}^{(3-n) / 2}\right)\right\|^{\theta(p-1) / 2}\right) \leq M_{2},
$$

where $M_{1}, M_{2}$ depend only on $\left\|u ; L^{\infty}\left(\mathbb{R}, H^{1}\right)\right\|$. By Proposition 2.1 , one can partition $\mathbb{R}$ into a finite number of intervals such that

$$
M_{1}\left\|\rho ; L^{2}\left(I, \dot{H}^{(3-n) / 2}\right)\right\|^{\theta(p-1) / 2} \leq 1 / 2,
$$

and the number of intervals is also estimated in terms of $\left\|u ; L^{\infty}\left(\mathbb{R}, H^{1}\right)\right\|$. This yields an estimate of $\left\|u ; X^{1}(I)\right\|$ for each such interval. Furthermore $u \in X^{1}(\mathbb{R})$ and $\left\|u ; X^{1}(\mathbb{R})\right\|$ is estimated by a (computable) power of $\left\|u ; L^{\infty}\left(\mathbb{R}, H^{1}\right)\right\|$. This completes the proof for $n \geq 2$.

REMARK 3.2. For $n=2,3$, the argument is the same whether one uses $u$ or $\rho$. For $n \geq 4$, the argument can also be made by using $\rho$ and the fact that

$$
\left\|\rho ; \dot{H}_{n /(n-\sigma)}^{\sigma}\right\| \leq C\left\|u ; \dot{H}^{\sigma}\right\|^{2}
$$

for $0 \leq \sigma \leq 1$ by Leibniz and Sobolev inequalities. Again one ends up with the condition (3.14), however with

$$
\sigma_{0}=2 \sigma_{c}(n-3) /\left(2 n-5-4 \sigma_{c}\right)
$$

which makes the restriction on $\sigma$ slightly stronger.

The case $\mathbf{n}=\mathbf{1}$. Here we work with $\rho$. For low values of $p$, we shall need the implication for $\rho$ of some Strichartz norms of $u$. We need the following lemma.

Lemma 3.1. Let $0 \leq \sigma<1 / r \leq 1 / 2$. Then

$$
\left\|\rho ; \dot{H}_{(2 / r-\sigma)^{-1}}^{\sigma}\right\| \leq C\left\|u ; \dot{H}_{r}^{\sigma}\right\|^{2},
$$

and therefore for $2 / q=\delta(r)$ and for any interval $I$

$$
\left\|\rho ; L^{q / 2}\left(I, \dot{H}_{(2 / r-\sigma)^{-1}}^{\sigma}\right)\right\| \leq C\left\|u ; L^{q}\left(I, \dot{H}_{r}^{\sigma}\right)\right\|^{2} .
$$

Proof of Lemma 3.1. We estimate by fractional Leibniz and Sobolev inequalities

$$
\left\|\omega^{\sigma} \rho\right\|_{(2 / r-\sigma)^{-1}} \leq C\left\|\omega^{\sigma} u\right\|_{r}\|u\|_{(1 / r-\sigma)^{-1}} \leq C\left\|\omega^{\sigma} u\right\|_{r}^{2} .
$$

We come back to the proof of the proposition. We start again from (3.4), so that we need to estimate $u$ in $L^{k}\left(I, L^{\ell}\right)$ with

$$
\left\{\begin{array}{l}
2 / k=\left(1 / 2-\sigma_{c}\right)(1-\delta), \\
1 / \ell=\left(1 / 2-\sigma_{c}\right) \delta
\end{array}\right.
$$


for some $\delta$ with $0 \leq \delta \leq 1 / 2$, or equivalently with

$$
\begin{gathered}
2 / k+1 / \ell=1 / 2-\sigma_{c}, \\
0 \leq 1 / \ell \leq\left(1 / 2-\sigma_{c}\right) / 2 .
\end{gathered}
$$

We estimate

$$
\begin{aligned}
\left\|u ; L^{k}\left(I, L^{\ell}\right)\right\|^{2} & =\left\|\rho ; L^{k / 2}\left(I, L^{\ell / 2}\right)\right\| \\
& \leq C\left\|\rho ; L^{2}\left(I, \dot{H}^{1}\right)\right\|^{\theta}\left\|\rho ; L^{q / 2}\left(I, \dot{H}_{(2 / r-\sigma)^{-1}}^{\sigma}\right)\right\|^{1-\theta}
\end{aligned}
$$

by Sobolev inequalities, for some $\sigma, \theta$ and admissible $(q, r)$ satisfying $0 \leq \sigma<1 / r \leq 1 / 2$, $0<\theta \leq 1$ and

$$
\left\{\begin{array}{l}
2 / k=\theta / 2+(1-\theta) 2 / q, \\
1 / \ell=-\theta / 4+(1-\theta)(1 / r-\sigma) .
\end{array}\right.
$$

Substituting (3.26) into (3.23) and (3.24) yields

$$
\begin{gathered}
\sigma_{c}=\theta / 4+(1-\theta) \sigma, \\
0 \leq-\theta / 4+(1-\theta)(1 / r-\sigma) \leq\left(1 / 2-\sigma_{c}\right) / 2 .
\end{gathered}
$$

For $\sigma_{c}=1 / 4$, namely $p=9$, we must take $\sigma=1 / 4$, and we ensure (3.27) and (3.28) by taking $r=2$ and $\theta=1 / 2$.

For $\sigma_{c} \neq 1 / 4$, we must take

$$
0 \leq \sigma<\sigma_{c}<1 / 4 \quad \text { or } \quad 1 / 4<\sigma_{c}<\sigma<1 / 2,
$$

and the elimination of $\theta$ between (3.27) and (3.28) yields

$$
\sigma_{c} \leq \frac{\left(4 \sigma_{c}-1\right)}{(4 \sigma-1) r} \leq\left(1+2 \sigma_{c}\right) / 4
$$

which implies the condition $\sigma<1 / r$ since

$$
\frac{1}{r} \geq \frac{4 \sigma-1}{4 \sigma_{c}-1} \sigma_{c}=\sigma+\frac{\sigma-\sigma_{c}}{4 \sigma_{c}-1}>\sigma
$$

by (3.29). One can fulfill (3.30) with $r=2$ provided

$$
\sigma_{+} \gtrless \sigma \gtrless \sigma_{-}\left(\gtrless \sigma_{c}\right) \gtrless 1 / 4,
$$

where

$$
\sigma_{+}=\left(6 \sigma_{c}-1\right) / 8 \sigma_{c}, \quad \sigma_{-}=\left(10 \sigma_{c}-1\right) /\left(8 \sigma_{c}+4\right),
$$

which is compatible with (3.29) provided $\sigma_{-} \geq 0$, namely $\sigma_{c} \geq 1 / 10$ or $p \geq 6$. In that case we take $r=2$ and we can take

$$
\begin{cases}0 \leq \sigma \leq \sigma_{-}\left(<\sigma_{c}\right) & \text { for } 1 / 10 \leq \sigma_{c} \leq 1 / 6, \\ (0 \leq) \sigma_{+} \leq \sigma \leq \sigma_{-}\left(<\sigma_{c}\right) & \text { for } 1 / 6 \leq \sigma_{c}<1 / 4 \\ \sigma_{+}=\sigma=\sigma_{-}=\sigma_{c}=1 / 4 & \text { for } \sigma_{c}=1 / 4 \\ \left(\sigma_{c}<\right) \sigma_{-} \leq \sigma \leq \sigma_{+}(<1 / 2) & \text { for } 1 / 4<\sigma_{c}<1 / 2\end{cases}
$$


with $\theta$ defined by (3.27) for $\sigma_{c} \neq 1 / 4$. For such $(\sigma, \theta)$, one obtains

$$
\left\|u ; L^{k}\left(I, L^{r}\right)\right\| \leq C\left\|\rho ; L^{2}\left(I, \dot{H}^{1}\right)\right\|^{\theta / 2}\left\|u ; L^{\infty}\left(I, \dot{H}^{\sigma}\right)\right\|^{1-\theta}
$$

which implies (3.16) with $n=1$.

For $0<\sigma_{c}<1 / 10$, namely $5<p<6$, one can take $\sigma=0$ and take for $r$ the minimal value allowed by (3.30), namely

$$
4 / r=\left(1+2 \sigma_{c}\right) /\left(1-4 \sigma_{c}\right)
$$

and $\theta=4 \sigma_{c}$. One then obtains

$$
\left\|u ; L^{k}\left(I, L^{\ell}\right)\right\| \leq C\left\|\rho ; L^{2}\left(I, \dot{H}^{1}\right)\right\|^{\theta / 2}\|u ; X(I)\|^{1-\theta}
$$

so that by (3.4)

$$
\begin{aligned}
\left\|u ; X^{1}(I)\right\| \leq & C\left(\left\|u ; L^{\infty}\left(I, H^{1}\right)\right\|+\left\|\rho ; L^{2}\left(I, \dot{H}^{1}\right)\right\|^{(p-1) \theta / 2}\right. \\
& \left.\times\left\|u ; X^{1}(I)\right\|^{1+(p-1)(1-\theta)}\right),
\end{aligned}
$$

which again gives an estimate of $\left\|u, X^{1}(I)\right\|$ provided $\left\|\rho ; L^{2}\left(I, \dot{H}^{1}\right)\right\|$ is sufficiently small.

The end of the proof proceeds as in the case $n \geq 2$.

REMARK 3.3. If one wants to use values of $\sigma$ arbitrarily close to $\sigma_{c}$ for $\sigma_{c}>1 / 4$, one needs to take $r>2$ in the region $\sigma_{c}<\sigma<\sigma_{-}$. The lowest possible value of $r$ is given by (see (3.30))

$$
4 / r=\left(1+2 \sigma_{c}\right)(4 \sigma-1) /\left(4 \sigma_{c}-1\right) .
$$

REMARK 3.4. One could also use $u$ instead of $\rho$ in the case $n=1$. From the inequality

$$
\rho^{3 / 2} \leq(3 / 4) \int d x \rho^{1 / 2}\left|\rho^{\prime}\right| \leq(3 / 4)\|\rho\|_{1}^{1 / 2}\left\|\rho^{\prime}\right\|_{2}
$$

we obtain

$$
\left\|u ; L^{6}\left(\mathbb{R}, L^{\infty}\right)\right\|^{3}=\left\|\rho ; L^{3}\left(\mathbb{R}, L^{\infty}\right)\right\|^{3 / 2} \leq(3 / 4)\|u\|_{2}\left\|\rho ; L^{2}\left(\mathbb{R}, \dot{H}^{1}\right)\right\|
$$

and one can perform the estimates by using $\left\|u ; L^{6}\left(\mathbb{R}, L^{\infty}\right)\right\|$ instead of $\left\|\rho: L^{2}\left(\mathbb{R}, \dot{H}^{1}\right)\right\|$. The results are essentially the same with, however, stronger restrictions on $\sigma$.

We now exploit Proposition 3.1 to prove AC in $H^{1}$ for the NLS equation (1.1) with nonlinearity (3.1). We first recall some standard results on scattering for that equation [2].

Proposition 3.2. Let $0 \leq \sigma_{c}<1, \sigma_{c}<1 / 2$ for $n=1$, or equivalently $p \geq 1+4 / n$, $p<1+4 /(n-2)$ for $n \geq 3$, and $\lambda>0$.

(1) Let $u_{+} \in H^{1}$. Then the NLS equation (1.1), (3.1) has a unique solution $u \in$ $X_{l o c}^{1}(\mathbb{R}) \cap X^{1}\left(\mathbb{R}^{+}\right)$such that

$$
\left\|U(-t) u(t)-u_{+} ; H^{1}\right\| \rightarrow 0
$$

when $t \rightarrow \infty$.

(2) Let $u \in X^{1}\left(\mathbb{R}^{+}\right)$be a solution. Then there exists $u_{+} \in H^{1}$ such that (3.41) holds. 
Sketch of proof. The proof uses mainly Strichartz inequalities. In order to prove part (1), one starts from the integral equation (3.3) with $u_{+}=U\left(-t_{0}\right) u_{0}$ and $t_{0} \rightarrow \infty$, namely

$$
u(t)=U(t) u_{+}+i \int_{t}^{\infty} d t^{\prime} U\left(t-t^{\prime}\right) g\left(\rho\left(t^{\prime}\right)\right) u\left(t^{\prime}\right),
$$

and one solves that equation locally in a neighborhood of infinity in time, namely in $I=[T, \infty)$ for $T$ sufficiently large. The proof uses the estimate (3.4) followed by

$$
\left\|u ; L^{k}\left(I, L^{\ell}\right)\right\| \leq C\left\|u ; L^{q_{1}}\left(I, \dot{H}_{r_{1}}^{\sigma_{c}}\right)\right\| \leq C\left\|u ; X^{1}(I)\right\|
$$

for admissible $\left(q_{1}, r_{1}\right)$ with $k=q_{1}<\infty$, namely

$$
0<2 / k=\left(n / 2-\sigma_{c}\right)(1-\delta)=2 / q_{1}=\delta\left(r_{1}\right)
$$

which can always be realized for suitable $\delta$.

In order to prove part (2), one estimates

$$
\begin{gathered}
\left\|U\left(-t_{1}\right) u\left(t_{1}\right)-U\left(-t_{2}\right) u\left(t_{2}\right) ; H^{1}\right\|=\left\|\int_{t_{1}}^{t_{2}} d t^{\prime} U\left(t-t^{\prime}\right) g\left(\rho\left(t^{\prime}\right)\right) u\left(t^{\prime}\right) ; H^{1}\right\| \\
\leq C\left\|u ; X^{1}(I)\right\|\left\|u ; L^{q_{1}}\left(I, \dot{H}_{r_{1}}^{\sigma_{c}}\right)\right\|^{p-1}
\end{gathered}
$$

with $I=\left[t_{1}, t_{2}\right]$, and the last norm tends to zero when $t_{1}, t_{2} \rightarrow \infty$ for $u \in X^{1}\left(\mathbb{R}^{+}\right)$and $q_{1}<\infty$.

The previous proposition yields the existence of the wave operators in part (1) and the fact that $\mathrm{AC}$ holds for solutions in $X^{1}(\mathbb{R})$ in part (2). Putting together Propositions 3.1 and 3.2 yields $\mathrm{AC}$ for finite energy solutions.

Proposition 3.3. Let $0<\sigma_{c}<1, \sigma_{c}<1 / 2$ for $n=1$, or equivalently $p>1+4 / n$, $p<1+4 /(n-2)$ for $n \geq 3$, and let $\lambda>0$. Let $u$ be a finite energy solution of the NLS equation (1.1), (3.1), namely a solution $u \in X_{l o c}^{1}(\mathbb{R})$. Then $u \in X^{1}(\mathbb{R})$ and there exist $u_{ \pm} \in H^{1}$ such that

$$
\left\|U(-t) u(t)-u_{ \pm} ; H^{1}\right\| \rightarrow 0
$$

when $t \rightarrow \pm \infty$.

We now turn to the Hartree equation (1.1) with $g$ given by (1.3). We assume that $V \in L^{p}$ for suitable $p$, for which we shall use the parameter $\sigma_{c}$ defined by

$$
\sigma_{c}=n / 2 p-1 .
$$

The treatment extends in a trivial way to more general $V$ such as those considered in Proposition 2.2.

The main technical result is the following proposition.

Proposition 3.4. Let $n \geq 3$. Let $0<\sigma_{c}<1, \sigma_{c} \leq 1 / 2$ for $n=3$, or equivalently $n / 4<p<n / 2, p \geq 1$ for $n=3$. Let $V \in L^{p}$ be real even and let $u \in X_{\text {loc }}^{1}(\mathbb{R}) \cap L^{\infty}\left(\mathbb{R}, H^{1}\right)$ be a solution of the Hartree equation (1.1), (1.3) such that $\rho=|u|^{2} \in L^{2}\left(\mathbb{R}, \dot{H}^{(3-n) / 2}\right)$. Then $u \in X^{1}(\mathbb{R})$.

REMARK 3.5. For nonnegative $V$, the Cauchy problem is globally well posed in $H^{1}$ and yields solutions $u \in X_{l o c}^{1}(\mathbb{R}) \cap L^{\infty}\left(\mathbb{R}, H^{1}\right)$. 
Sketch of proof. The proof follows the same pattern as that of Proposition 3.1. We start again from (3.3). Using the Strichartz estimates and the Young inequality, we estimate

$$
\left\|u ; X^{1}(I)\right\| \leq C\left(\left\|u_{0} ; H^{1}\right\|+\|V\|_{p}\left\|u ; X^{1}(I)\right\|\left\|u ; L^{k}\left(I, L^{\ell}\right)\right\|^{2}\right),
$$

where now

$$
\left\{\begin{array}{l}
2 / k=1-\delta, \\
n / \ell=n / 2-\sigma_{c}+\delta-1
\end{array}\right.
$$

for some $\delta, 0 \leq \delta \leq 1$. It is then sufficient to estimate $u \in L^{k}\left(I, L^{\ell}\right)$ for $0<2 / k \leq 1$ and

$$
2 / k+n / \ell=n / 2-\sigma_{c} .
$$

The proof then proceeds as for the NLS equation. In particular one uses the estimate (3.8) with $k, \ell$ satisfying (3.9) so that $0<\theta=2(1-\delta) \leq 1$, which ensures the condition $0<2 / k \leq 1$.

The analogue of Proposition 3.2 can be proved for the Hartree equation [13], and the final result follows therefrom and from Propositions 3.4 and 2.2.

Proposition 3.5. Let $n \geq 3$. Let $0<\sigma_{c}<1, \sigma_{c} \leq 1 / 2$ for $n=3$, or equivalently $n / 4<p<n / 2, p \geq 1$ for $n=3$. Let $V \in L^{p}$ be real radial and nonincreasing (and therefore nonnegative). Let $u$ be a finite energy solution of the Hartree equation (1.1), (1.3), namely a solution $u \in X_{l o c}^{1}(\mathbb{R})$. Then $u \in X^{1}(\mathbb{R})$ and there exist $u_{ \pm} \in H^{1}$ satisfying (3.45) when $t \rightarrow \pm \infty$.

\section{Appendix 1. Estimate of the RHS of (2.21) in $\mathbf{H}^{1 / 2}$ for $h=|x|$.} LEMMA.

$$
|\langle\rho, \nabla|x| \star \operatorname{Im} \bar{u} \nabla u\rangle| \leq C\|u\|_{2}^{2}\left\|u ; \dot{H}^{1 / 2}\right\|^{2} .
$$

Proof. We estimate

$$
\begin{aligned}
& |\langle\rho, \nabla|x| \star \operatorname{Im} \bar{u} \nabla u\rangle| \leq|\langle\nabla u, u(\nabla|x| \star \rho)\rangle| \\
& \leq\left\|\omega^{1 / 2} u\right\|_{2}\left\|\omega^{1 / 2} u(\nabla|x| \star \rho)\right\|_{2} \\
& \leq C\left(\left\|\omega^{1 / 2} u\right\|_{2}^{2}\|\nabla|x| \star \rho\|_{\infty}\right. \\
& \left.\quad+\left\|\omega^{1 / 2} u\right\|_{2}\|u\|_{r}\left\|\omega^{1 / 2}(\nabla|x| \star \rho)\right\|_{n / \delta}\right)
\end{aligned}
$$

with $\delta=\delta(r)>0$, by fractional Leibniz inequalities. Clearly

$$
\|\nabla|x| \star \rho\|_{\infty} \leq\|\rho\|_{1}
$$

We then use the fact that

$$
F(\nabla|x|)=C P \xi|\xi|^{-(n+1)},
$$

where $F$ is the Fourier transform and $P$ denotes the principal value ([22], Theorem 5, p. 73 with $k=1$ ) so that

$$
\omega^{1 / 2}(\nabla|x| \star \rho)=C F^{-1}\left(\xi|\xi|^{-(n+1 / 2)} \widehat{\rho}(\xi)\right)=C x|x|^{-3 / 2} \star \rho
$$


and therefore

$$
\left\|\omega^{1 / 2}(\nabla|x| \star \rho)\right\|_{n / \delta} \leq C\|\rho\|_{s / 2}=C\|u\|_{s}^{2}
$$

by the Hardy Littlewood Sobolev inequality ([22], Theorem 1, p. 119), where

$$
\delta+2 \delta(s)=1 / 2,
$$

provided $0<\delta<n$ and $0<\delta(s)<n / 2$. The last term in (A1.2) is then estimated by

$$
C\left\|\omega^{1 / 2} u\right\|_{2}\|u\|_{r}\|u\|_{s}^{2} \leq C\left\|\omega^{1 / 2} u\right\|_{2}^{2}\|u\|_{2}^{2}
$$

by Sobolev inequalities, which together with (A1.2) and (A1.3) yields (A1.1). One can easily choose $r$ satisfying the required restrictions, for instance by taking $r=s$, which yields $\delta=1 / 6$.

Appendix 2. A quadratic identity for the NLKG equation. As mentioned in Section 2, the algebraic structure, (2.1) and (2.2), is realized for any system with symmetric conserved energy momentum tensor $T_{\lambda \mu}$, namely

$$
\left\{\begin{array}{l}
\partial_{t} \sigma+\nabla \cdot j=0, \\
\partial_{t} j+\nabla \cdot T=0
\end{array}\right.
$$

where $\sigma=T_{00}$ is the energy density, $j_{k}=-T_{0 k}=-T_{k 0}$ is both the energy current and the momentum density, and $T=\left\{T_{k \ell}\right\}$ is the space-space part of $T_{\lambda \mu}$. Here we use $\sigma$ instead of $\rho$ in order to keep the notation $\rho$ for $|u|^{2}$, greek (resp. latin) indices run from 0 to $n$ (resp. from 1 to $n$ ), and the index 0 refers to time. We shall also need the Minkowski metric $\eta_{\lambda \mu}$ with $\eta_{00}=-\eta_{j j}=1$. We now consider the NLKG (or nonlinear wave NLW) equation

$$
\square u+g(\rho) u=0,
$$

where $g=g(\rho)$ is a real function of $\rho=|u|^{2}$. The Lagrangian density is

$$
L(u)=\left|\partial_{t} u\right|^{2}-|\nabla u|^{2}-G(\rho),
$$

with $G$ defined by (2.9). The energy momentum tensor is well known to be

$$
T_{\lambda \mu}=2 \operatorname{Re} \partial_{\lambda} \bar{u} \partial_{\mu} u-\eta_{\lambda \mu} L .
$$

We define as before for real even $h$

$$
J=(1 / 2)\langle\sigma, h \star \sigma\rangle
$$

so that

$$
\begin{gathered}
M \equiv \partial_{t} J=-\langle\sigma, \nabla h \star j\rangle, \\
\partial_{t} M=\partial_{t}^{2} J=-\left\langle j, \nabla^{2} h \star j\right\rangle+\left\langle\sigma, \nabla^{2} h \star T\right\rangle .
\end{gathered}
$$

Substituting $\sigma=T_{00}, j_{k}=-T_{0 k}$ and $T=\left\{T_{k \ell}\right\}$ given by (A2.4) into (A2.7) yields

$$
\begin{gathered}
\partial_{t} M=-\left\langle 2 \operatorname{Re} \nabla \bar{u} \partial_{t} u, \nabla^{2} h \star 2 \operatorname{Re} \nabla \bar{u} \partial_{t} u\right\rangle \\
+\left\langle\left|\partial_{t} u\right|^{2}+|\nabla u|^{2}+G, \Delta h \star\left(\left|\partial_{t} u\right|^{2}-|\nabla u|^{2}-G\right)+\nabla^{2} h \star 2 \nabla \bar{u} \nabla u\right\rangle,
\end{gathered}
$$


and finally, ordering the terms by the powers of $G$,

$$
\begin{aligned}
\partial_{t} M= & -\langle G, \Delta h \star G\rangle+\left\langle G,-2 \Delta h \star|\nabla u|^{2}+\nabla^{2} h \star 2 \nabla \bar{u} \nabla u\right\rangle \\
& +\left\langle\left|\partial_{t} u\right|^{2}+|\nabla u|^{2}, \Delta h \star\left(\left|\partial_{t} u\right|^{2}-|\nabla u|^{2}\right)+\nabla^{2} h \star 2 \nabla \bar{u} \nabla u\right\rangle \\
& -\left\langle 2 \operatorname{Re} \nabla \bar{u} \partial_{t} u, \nabla^{2} h \star 2 \operatorname{Re} \nabla \bar{u} \partial_{t} u\right\rangle .
\end{aligned}
$$

In space dimension $n=1$, the linear term in $G$ vanishes and (A2.8) reduces to

$$
\begin{aligned}
\partial_{t} M & =-\left\langle G, h^{\prime \prime} \star G\right\rangle+\left\langle\left|\partial_{t} u\right|^{2}+\left|\partial_{x} u\right|^{2}, h^{\prime \prime} \star\left(\left|\partial_{t} u\right|^{2}+\left|\partial_{x} u\right|^{2}\right\rangle\right. \\
& -\left\langle 2 \operatorname{Re} \partial_{x} \bar{u} \partial_{t} u, h^{\prime \prime} \star 2 \operatorname{Re} \partial_{x} \bar{u} \partial_{t} u\right\rangle \\
& =\left\langle\left|\partial_{t} u-\partial_{x} u\right|^{2}, h^{\prime \prime} \star\left|\partial_{t} u+\partial_{x} u\right|^{2}\right\rangle-\left\langle G, h^{\prime \prime} \star G\right\rangle
\end{aligned}
$$

so that if $h^{\prime \prime}$ has a given sign, the kinetic and potential terms have opposite signs, which precludes a straightforward use of that identity to derive estimates.

Appendix 3. Relation between the original and the quadratic Morawetz identities. Here we rewrite the original Morawetz identity for the NLS equation in a form which exhibits its relation to the quadratic identity derived in Section 2. The original version starts from the quantity $\langle\nabla h, j\rangle$ with $j=\operatorname{Im} \bar{u} \nabla u$. Using a space translate instead of $\nabla h$ is then equivalent to considering the quantity

$$
M_{0}(x)=-\nabla h \star j
$$

so that $M$ defined in (2.4) is simply $M=\left\langle\rho, M_{0}\right\rangle$. Using the evolution equation of $j$ given by (2.2) and (2.14) yields

$$
\begin{aligned}
\partial_{t} M_{0} & =-\nabla^{2} h \star T \\
& =\nabla^{2} h \star(\nabla \bar{u} \nabla u)+\Delta h \star(-(1 / 4) \Delta \rho+\rho g-G) .
\end{aligned}
$$

For $h(x)=|x|$ and by the use of (2.22), we obtain

$$
\partial_{t} M_{0}=\nabla^{2} h \star(\nabla \bar{u} \nabla u)-(1 / 4) \Delta^{2}|x| \star \rho+(n-1)|x|^{-1} \star(\rho g-G)
$$

for $n>1$, which is the original Morawetz identity for NLS. The first term on the RHS is nonnegative. The second term is positive for $n \geq 3$ only since

$$
\Delta^{2}|x|= \begin{cases}-8 \pi \delta(x) & \text { for } n=3, \\ -(n-1)(n-3)|x|^{-3} & \text { for } n \geq 4 .\end{cases}
$$

Early applications of the method [12], 15] used (A3.3) to derive an estimate of the last term and were therefore restricted to space dimension $n \geq 3$. Taking the scalar product of $M_{0}$ with $\rho$ gives a useful quantity because $\rho$ satisfies the conservation law (2.1). 
Appendix 4. Proof of (2.21) by regularization. Let $u \in X_{l o c}^{1}(I)$ be a solution of the NLS equation (2.7) and let $\varphi \in \mathscr{C}_{0}^{\infty}\left(\mathbb{R}^{n}, \mathbb{R}^{+}\right)$with $\|\varphi\|_{1}=1$ denote a regularizing function of the space variable which will eventually converge to the measure $\delta$. We define $u_{\varphi}=\varphi \star u, \rho_{\varphi}=\left|u_{\varphi}\right|^{2}, j_{\varphi}=\operatorname{Im} \bar{u}_{\varphi} \nabla u_{\varphi}, f(u)=g\left(|u|^{2}\right) u, f_{\varphi}=\varphi \star f(u)$ and $f_{\neq}=f_{\varphi}-f\left(u_{\varphi}\right)$. Then $u_{\varphi} \in \bigcap_{\sigma \geq 0} \mathscr{C}\left(I, H^{\sigma}\right)$, and $u_{\varphi}$ satisfies the equation

$$
i \partial_{t} u_{\varphi}=-(1 / 2) \Delta u_{\varphi}+f_{\varphi},
$$

which is the regularized form of (2.7). From (A4.1), we obtain the regularized form of (2.1) and (2.2) by direct computation. Namely

$$
\begin{gathered}
\partial_{t} \rho_{\varphi}+\nabla \cdot j_{\varphi}=P_{1}, \\
\partial_{t} j_{\varphi}+\nabla \cdot T\left(u_{\varphi}\right)=P_{2}
\end{gathered}
$$

where

$$
\begin{gathered}
P_{1}=2 \operatorname{Im}\left(\bar{u}_{\varphi} f_{\neq}\right), \\
T_{k \ell}\left(u_{\varphi}\right)=\operatorname{Re} \nabla_{k} \bar{u}_{\varphi} \nabla_{\ell} u_{\varphi}-\delta_{k \ell}\left((1 / 4) \Delta \rho_{\varphi}-\rho_{\varphi} g\left(\rho_{\varphi}\right)+G\left(\rho_{\varphi}\right)\right), \\
P_{2}=\operatorname{Re}\left(f_{\neq} \nabla \bar{u}_{\varphi}-\bar{u}_{\varphi} \nabla f_{\neq}\right) .
\end{gathered}
$$

Using (A4.2) and (A4.3) and the regularity properties of $u_{\varphi}$, we can derive the regularized version of (2.21) in the same way as in Section 2 (see (2.16)-(2.20)). Namely

$$
\begin{gathered}
\int_{t_{1}}^{t_{2}} d t\left\{(1 / 2)\left\langle\nabla \rho_{\varphi}, \Delta h \star \nabla \rho_{\varphi}\right\rangle+\left\langle\rho_{\varphi}, \Delta h \star\left(\rho_{\varphi} g\left(\rho_{\varphi}\right)-G\left(\rho_{\varphi}\right)\right)\right\rangle\right. \\
\left.+R\left(u_{\varphi}\right)-S_{\varphi}\right\}=-\left.\left\langle\rho_{\varphi}, \nabla h \star j_{\varphi}\right\rangle\right|_{t_{1}} ^{t_{2}},
\end{gathered}
$$

where

$$
\begin{aligned}
R(v) & =\left\langle\bar{v} v, \nabla^{2} h \star \nabla \bar{v} \nabla v\right\rangle-\left\langle\bar{v} \nabla v, \nabla^{2} h \star \bar{v} \nabla v\right\rangle, \\
S_{\varphi} & =-\left\langle j_{\varphi}, \nabla h \star P_{1}\right\rangle+\left\langle\rho_{\varphi}, \nabla h \star P_{2}\right\rangle \\
& =2 \operatorname{Re}\left\{\left\langle u_{\varphi} \nabla \bar{u}_{\varphi}, \nabla h \star \bar{u}_{\varphi} f_{\neq}\right\rangle+\left\langle\rho_{\varphi}, \nabla h \star f_{\neq} \nabla \bar{u}_{\varphi}\right\rangle\right\}
\end{aligned}
$$

by a straightforward rewriting.

We now remove the cutoff, and without loss of generality we restrict our attention to the case of a single power interaction of the form (3.1). We restrict ourselves to proving that

$$
\lim _{\varphi \rightarrow \delta} \int_{t_{1}}^{t_{2}} d t S_{\varphi}=0
$$

since the remaining terms in (A4.7) converge to the corresponding terms without $\varphi$ by estimates similar to (2.29) and (2.31). We estimate

$$
\left|S_{\varphi}\right| \leq 4\|u\|_{2}^{2}\|\nabla u\|_{2}\left\|f_{\neq}\right\|_{2}
$$

since $\|\nabla h\|_{\infty}=1$ by (2.22). From the identity

$$
f_{\neq}=\varphi \star f(u)-f(u)+f(u)-f\left(u_{\varphi}\right)
$$

and from the estimates

$$
|f(u)| \leq C|u|^{p},\left|f(u)-f\left(u_{\varphi}\right)\right| \leq C\left|u-u_{\varphi}\right|\left(|u|^{p-1}+\left|u_{\varphi}\right|^{p-1}\right),
$$


we obtain

$$
\left\|f_{\neq}\right\|_{2} \leq\|\varphi \star f(u)-f(u)\|_{2}+C\|u\|_{2 p}^{p-1}\left\|u-u_{\varphi}\right\|_{2 p} .
$$

We next estimate $\|u\|_{2 p}$. For $n=1,2$ and for $n \geq 3$ with $p-1 \leq 2 /(n-2)$, we estimate

$$
\|u\|_{2 p} \leq C\left\|u ; H^{1}\right\| \in L_{l o c}^{\infty}(I) .
$$

For $n \geq 3$ and

$$
2 /(n-2)<p-1<4 /(n-2)
$$

we estimate

$$
\|u\|_{2 p} \leq C\left\|u ; \dot{H}_{r}^{1}\right\| \in L_{l o c}^{q}(I)
$$

where

$$
0<2 / q=\delta(r)=\delta(2 p)-1<(n-2) /(n+2),
$$

so that $\|f(u)\|_{2}=C\|u\|_{2 p}^{p} \in L_{l o c}^{k}(I)$ with

$$
0<2 / k=(p-1)(n / 2-1)-1<1 .
$$

In both cases $\left\|f_{\neq}\right\|_{2}$ tends to zero for each $t \in I$ when $\varphi$ tends to $\delta$, and (A4.10) follows from (A4.11) and from the Lebesgue dominated convergence theorem.

\section{REFERENCES}

[1] J. Bourgain, Global solutions of non linear Schrödinger equations, AMS Colloq. Pub. 46, 1999.

[2] T. Cazenave, Semilinear Schrödinger equations, Courant Lecture Notes in Math. 10 AMS, 2003. MR 2002047 (2004j:35266)

[3] J. Colliander, M. Grillakis and N. Tzirakis, Improved interaction Morawetz inequalities for the cubic nonlinear Schrödinger equation on $\mathbb{R}^{2}$, Int. Math. Res. Not. IMRN 2007, no. 23, Art. ID mm090, 30pp. MR.2377216

[4] J. Colliander, M. Grillakis and N. Tzirakis, Tensor products and correlation estimates with applications to nonlinear Schrödinger equations, Preprint, math.AP/0807.0871.

[5] J. Colliander, H. Holmer, M. Visan and X. Zhang, Global existence and scattering for rough solutions to generalized nonlinear Schrödinger equations on $\mathbb{R}$, Commun. Pure Appl. Anal. 7 (2008), 467-489. MR 2379437 (2009c:35433)

[6] J. Colliander, M. Keel, G. Staffilani, H. Takaoka and T. Tao, Global well-posedness and scattering in the energy space for the critical nonlinear Schrödinger equation in $\mathbb{R}^{3}$, Annals Math. 166 (2007), 1-100. MR2359021

[7] J. Colliander, M. Keel, G. Staffilani, H. Takaoka and T. Tao, Global existence and scattering for rough solutions to a nonlinear Schrödinger equation on $\mathbb{R}^{3}$, Comm. Pure Appl. Math. 57 (2004) no. 8, 987-1014. MR2053757 (2005b:35257)

[8] D. De Silva, N. Pavlovic, G. Staffilani and N. Tzirakis, Global well-posedness and polynomial bounds for the defocusing $L^{2}$-critical nonlinear Schrödinger equation in higher dimensions, Commun. Pure Appl. Anal. 6 (2007), 1023-1041. MR 2341818

[9] D. De Silva, N. Pavlovic, G. Staffilani and N. Tzirakis, Global well-posedness and polynomial bounds for the defocusing $L^{2}$-critical nonlinear Schrödinger equation in $\mathbb{R}$, Comm. Partial Differential Equations 33 (2008), no. 7-9, 1395-1429. MR2450163

[10] Y. Fang and M. Grillakis, On the global existence of rough solutions to the cubic defocusing Schrödinger equation in $\mathbb{R}^{2+1}$, J. Hyp. Diff. Eq. 4 (2007), 233-257. MR2329384 (2008c:35303)

[11] D. Fang and Z. Han, The nonlinear Schrödinger equations with combined nonlinearities of powertype and Hartree-type, Preprint, math.AP/0808.1622.

[12] J. Ginibre and G. Velo, Scattering theory in the energy space for a class of nonlinear Schrödinger equations, J. Math. Pure Appl. 64 (1985), 363-401. MR839728 (87i:35171)

[13] J. Ginibre and G. Velo, Scattering theory in the energy space for a class of Hartree equations, in Nonlinear Wave Equations, Y. Guo Ed., Contemporary Mathematics 263, AMS 2000. MR 1777634 (2001g:35205) 
[14] J. Holmer and N. Tzirakis, Asymptotically linear solutions in $H^{1}$ of the 2D defocusing nonlinear Schrödinger and Hartree equations, Preprint, math.AP/0805.2925.

[15] J. Lin and W. Strauss, Decay and scattering of solutions of a nonlinear Schrödinger equation, J. Funct. Anal. 30 (1978), 245-263. MR.515228 (80k:35056)

[16] C. Miao, G. Xu and L. Zhao, Global wellposedness and scattering for the defocusing $H^{1 / 2}$ subcritical Hartree equation in $\mathbb{R}^{d}$, Preprint, math.AP/0805.3378.

[17] C. Morawetz, Time decay for the nonlinear Klein-Gordon equation, Proc. Roy. Soc. A206 (1968), 291-296. MR0234136 (38:2455)

[18] C. Morawetz and W. Strauss, Decay and scattering of solutions of a nonlinear relativistic wave equation, Comm. Pure Appl. Math. 25 (1972), 1-31. MR0303097 (46:2239)

[19] K. Nakanishi, Energy scattering for nonlinear Klein-Gordon and Schrödinger equations in spatial dimensions 1 and 2, J. Funct. Anal. 169 (1999), 201-225. MR.1726753 (2000m:35141)

[20] K. Nakanishi, Energy scattering for Hartree equations, Math. Res. Lett. 6 (1999), no. 1, 107-118. MR 1682697 (2000d:35173)

[21] F. Planchon and L. Vega, Bilinear virial identities and applications, Preprint, math.AP/0712.4076. To appear in Ann. Scient. ENS.

[22] E. M. Stein, Singular integrals and differentiability properties of functions, Princeton Univ. Press, 1967. MR0290095 (44:7280)

[23] T. Tao, Nonlinear dispersive equations, local and global analysis, Regional. Conf. Ser. Math. 106, AMS 2006. MR 2233925 (2008i:35211)

[24] T. Tao, M. Visan and X. Zhang, The nonlinear Schrödinger equation with combined power type non linearities, Comm. Partial Diff. Eq. 32 (2007), 1281-1343. MR2354495

[25] M. Visan and X. Zhang, Global wellposedness and scattering for a class of nonlinear Schrödinger equations below the energy space, Diff. Int. Eq. 22 (2009), 99-124. 